\title{
CCD SURVEY FOR WR STARS IN THE CENTRAL PART OF M33
}

\author{
L. DRISSEN ${ }^{1}$, A. F. J. MOFFAT ${ }^{2}$ and M. M. SHARA ${ }^{1}$ \\ ${ }^{1}$ Space Telescope Science Institute, Baltimore, MD (USA) \\ ${ }^{2}$ Département de physique, Université de Montréal, Montréal (CANADA)
}

There have been numerous surveys for Wolf-Rayet stars in M33 (among others: Wray and Corso 1972, Ap. J., 172, 577; Massey and Conti 1983, Ap. J., 273, 576; and Massey et al. 1987, P.A.S.P., 99, 816). About $100 \mathrm{WR}$ stars have been discovered and spectroscopically confirmed so far. All these surveys have used photographic plates and a relatively large $(\sim 100 \AA)$ filter centered on the $4640-4686 \AA$ emissionline region. This choice of filter does not favor the detection of the narrow-line WNL stars. We have thus obtained CCD images of the central $\left(8^{\prime} \times 20^{\prime}\right)$ part of M33 with a different set of filters (broad B and narrow $4686 / 35 \AA$ fwhm) with the $2.2 \mathrm{~m}$ telescope at KPNO and the $1.6 \mathrm{~m}$ telescope at Mont Mégantic in an attempt to detect new WR candidates. $\mathrm{R}$ and $\mathrm{H} \alpha$ images were also obtained.

Twenty-nine (29) new candidates have been found, half of them being of high quality. In the area surveyed, 43 spectroscopically confirmed WR stars were previously known. We have detected all but two of them (MC34 and MC45). This result shows that even if only our best candidates (detection level $\geq 7 \sigma$ ) are genuine WR stars, the incompleteness of previous surveys may be as high as $30 \%$. Since most of them are likely to be WNs, WC/WN ratios (and hence previous conclusions about the effects of metallicity and IMF gradients in this galaxy) are likely to be revised. Obviously, the WR content of M33 deserves further study.

Our $\mathrm{H} \alpha$ images allowed the detection of 15 ring nebulae around confirmed WR stars (details will be published elsewhere). Ring nebulae were also detected around 4 of our new WR candidates. WR ring nebulae in M33 are larger, on average, than their Galactic counterparts: while $80 \%$ of the Galactic rings are smaller than $20 \mathrm{pc}$, most of M33's WR rings are larger than 20 pc. This may be because the interstellar medium in M33 is more favorable to the expansion of such bubbles; but it could also be, at least in part, an observational effect, since we have probably missed all nebulae smaller than $5 \mathrm{pc}$. 